\title{
On Stiction Compensation Methods for Practical Nonlinear MPC Implementations
}

\author{
José Luis Pitarch \\ Systems Engineering and Automatic \\ Control Department. \\ Universidad de Valladolid. \\ Valladolid, Spain \\ jose.pitarch@autom.uva.es
}

\author{
Pedro Santos \\ Systems Engineering and Automatic \\ Control Department. \\ Universidad de Valladolid. \\ Valladolid, Spain \\ pedro.santos.bartolome@uva.es
}

\author{
César de Prada \\ Institute of Sustainable Processes (IPS) \\ Systems Engineering and Automatic Control \\ Department. Universidad de Valladolid \\ Valladolid, Spain \\ prada@autom.uva.es
}

\begin{abstract}
Stiction in actuators is a common issue within the process industry, which may considerably degrade the control performance in practice. In particular, the improved prediction capabilities of model predictive control (MPC) vanish if such actuator imperfections are neglected in actual implementations. In this work, the authors review the more recent approaches for stiction compensation in literature and propose two practical alternatives that are more suitable to use in large-scale nonlinear MPC problems. The proposals are illustrated and tested over a real industrial case study: the fiber humidity control in a medium-density fibreboard dryer.
\end{abstract}

\section{Keywords—stiction, MPC, windup, deadband, compensator}

\section{LiterATURE REVIEW \& MOTIVATION}

Although actuators are the most prevalent elements in a control loop, their behavior is often neglected when designing controllers. However, they are not perfect, making $u \neq u_{a}$ (see Fig. 1) in some situations, which contributes to a significant number of negative effects such as tracking issues, sustained closed-loop oscillations, etc. Among these practical limitations, saturation and rate constraints are always present in applications, being thus the type of actuator nonlinearities more successfully handled in both classical feedback and advanced MPCs. But there are also other undesirable issues such as stiction, backlash, deadband or hysteresis [1] [2] [3], that appear inherently to the actuators nature (flow valves, mechanical gears, electromagnetic devices, etc.) and are more difficult to account for in the control design phase [4]. In this paper we will focus on static friction or stiction, which traduces in a deadband behavior that can appear along the whole actuator range, and is especially harmful for MPC implementations in the process industry where the sampling time is in the scale of minutes.

The literature on stiction compensation is particularly broad, from reviews on methods [5][6] to applications [7]. Nevertheless, stiction compensation remains an active research topic, especially in MPC implementations [8]. From an extensive reading, one can conclude that, apart from exhaustive maintenance, the right way to handle actuator stiction is, by far, the use of a local control block between the main controller output and the actuator in a cascade fashion (see Fig. 1). This local compensator (linear or nonlinear) usually works at a higher frequency than the main controller, providing thus the necessary decoupling to design the main controller independently of

*Research supported by the European Union and the Spanish Government with project INOPTCON (MINECO/FEDER DPI2015-70975-P). actuators nonlinearities, i.e. presuming $u=u_{a}$. Examples of such kind of compensators are, e.g., PID-based [6][9], knockers [10], 2-move compensators [11] or constant reinforcement [12].

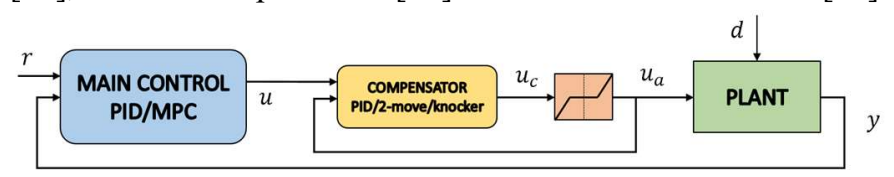

Figure 1. Usual cascade control to compensate actuators nonlinearities.

The above listed compensators belong to the so-called model-free methods which, roughly speaking, add compensating signals (piecewise constant, pulses, etc.) to the control ones, thereby reducing process variability at the price of increasing the actuator wear [13]. This may become an important limitation in practice because actuators can prematurely degrade. One can always choose a suitable tradeoff between actuator wear and control performance, by reducing the compensation-signal frequency, but this leads to additional issues to face: the assumption $u=u_{a}$ is no longer valid and the inner compensation loop dynamically modifies the overall plant behavior from the main controller point of view. Hence, re-tuning of both controllers is required to keep performance close to the ideal one, but no clear methodologies have been reported for this aim except in a few particular cases [14].

The alternative is using model-based methods to represent the actuator nonlinearities, which go from the naive ${ }^{1}[15]$

$$
u_{c}(t)= \begin{cases}u(t) & \text { if } \Delta u>\mathrm{f}_{\mathrm{s}} \mid \Delta u<-\mathrm{f}_{\mathrm{s}} \\ u(t-1) & \text { otherwise }\end{cases}
$$

to the more complex first-principles representations [6][16]. Given these models, the early proposal to the sticky actuator issue is to (approximately) invert the stiction nonlinearity in the compensator block so that $u_{a} \approx u$ at each time instant [4]. However, these solutions perform well only when $\Delta u$ is within the domain of perfect inversion, otherwise the system gets structural mismatch and the performance degrades [8].

The clear conclusion that can be extracted from the above review is that the better compensation, i.e. performance with minimum actuator wear, is obtained when the plant main controller is aware of the actuator nonlinearity. In this regard, the current trend is achieving stiction compensation through MPC, because of its natural way to handle input constraints [18]. Furthermore, in order to ease the numerical resolution, surro-

\footnotetext{
${ }^{1}$ Here $t$ represents the sampling instant, $\mathrm{f}_{\mathrm{s}}$ the highest force achieved due to static friction and $\Delta u=u(t)-u_{c}(t-1)$.
}

Copyright $\odot 2018$ IEEE. Personal use of this material is permitted. Permission from IEEE must be obtained for all other uses, in any current or future media, including reprinting/republishing this material for advertising or promotional purposes, creating new collective works, for resale or redistribution to servers or lists, or reuse of any copyrighted component of this work in other works. This is a preprint version which may contain errata. The published version is available in IEEE Xplore. DOI: 10.1109/ICSTCC.2018.8540748 
gate models of limited complexity for the actuator plus its possible local compensation strategy are added to the optimization [19]. Normally these solutions require that the MPC optimization problem is solved within small time grids, in order to properly represent the actuators dynamics, otherwise the MPC performance may still degrade. However, this requirement may impose an additional computational stress that is not really needed in systems where the main dynamics is slow, e.g. temperature processes where changing control inputs with frequencies lower than minutes makes no sense.

Including actuator nonlinearities like model (1) in the MPC can also be mathematically expressed by a series of if-then rules. This leads to mixed-integer programming (MIP) optimizations which combine continuous and discrete decisions. It is well-known that MIP problems considerably increase the computational complexity with respect to their smooth versions (i.e. without discrete decisions) which may mean a strong limitation for real-time implementations. Nevertheless, several works reported in the literature successfully followed this approach if the plant model is linear, leading to mixed-integer quadratic problems [20][21]. The issue with this approach comes from the main limitation of MPC itself: the predicted "optimal" performance deteriorates under strong plant-model mismatch. Thus, the above implementations may fail in situations where the system is not well described by the linearized model.

Therefore, two issues remain open for research: A) to avoid the use of MIP in real time and B) to consider more accurate nonlinear plant descriptions in the MPC. The first one has been partially addressed recently in [8], where the authors replace the hard discontinuities of the actuator model, i.e. (1), by an approximate stiction model which uses the hyperbolic tangent as smoothing function:

$$
\begin{gathered}
u_{c}(t)=\eta(t) \cdot u_{c}(t-1)+(1-\eta(t)) \cdot u(t) \\
\eta(t)=\frac{1}{2}\left(\tanh \left(\tau \cdot\left(\Delta u+\mathrm{f}_{\mathrm{s}}\right)\right)+\tanh \left(\tau \cdot\left(\mathrm{f}_{\mathrm{s}}-\Delta u\right)\right)\right)
\end{gathered}
$$

Here $\tau$ is the user-defined smoothing parameter to modify the sharpness, hence approximating the original model. It has been reported that the above model can exactly reproduce the actuator stiction for large enough values of $\tau$ but, for these values, constraints (2)-(3) are still too stiff to be effectively handled by gradient-based optimization solvers. Nonetheless, in the above cited reference the authors apparently got successful results using CasADi+IPOPT [22], but the academic example was a rather simple linear SISO system. Moreover, no comments on the extension/applicability of the proposed approach to multivariable MIMO and/or nonlinear systems were given. Indeed, we have tested this approach in our real case study (presented in next section) with no success due to:

- The resolution time for the nonlinear optimization exceeded by far the available time ( $<4 \mathrm{sec}$. in our case).

- The proposed "warm start" inspired in the 2-move compensation method with computation of the optimal steadystate input values did not provide accurate results in presence of disturbances and plant-model mismatch.

- The larger scale and nonlinear nature of the whole problem together with the stiff constraints (2)-(3) made the optimizer fall often into clearly suboptimal local minima.
These reasons motivated us to derive the practical approaches presented later in Section III, which partially address the above open issues and were suitable for the nonlinear MPC implementation in our case study: the medium density fibreboard (MDF) dryer, whose model details and control objectives are briefly outlined in the next section. Finally, some results from extensive simulation tests are provided in Section IV and a conclusions section close the paper, foreseeing further steps.

\section{CASE STUDY: THE MDF DRYER}

The industrial MDF dryer, represented in Fig. 2, is formed by: a) a mixture chamber that receives a flow of hot gasses from combustion and a cold one from the ambient, b) a thermally isolated $100 \mathrm{~m}$-long tube where the fiber comes in and the drying takes place, and c) a cyclone stage to separate fibers from gasses. The air used for drying is provided through the pressure gap created by a fan attached to the beginning of the tube dryer. The flows of hot and cold air are regulated by the opening of two flaps located on the air paths from the heatrecovery system $\left(a_{c}\right)$ and the ambient $\left(a_{f}\right)$. These two flaps are the manipulated variables for control during normal operation.

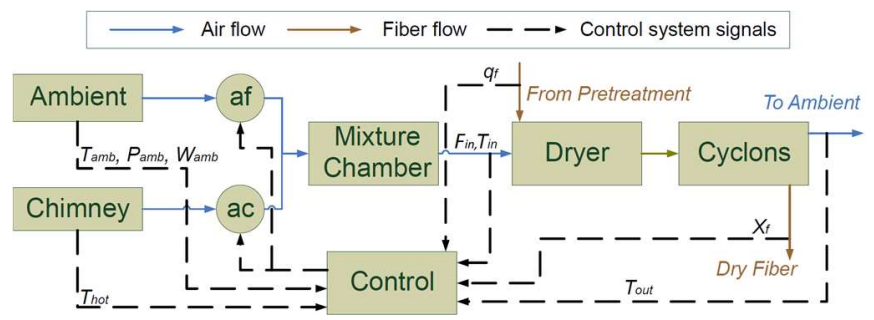

Figure 2. Plant diagram with its control architecture.

Temperatures of the hot gases inlet and the ambient are measured, as well as the environmental humidity. Air temperatures at the beginning and at the end of the tube dryer are also measured as well as the total air flow $F_{i n}$, inferred by a Pitot tube. The dry-fiber humidity $X_{f}$ is the target variable for control, as it significantly influences the rest of the MDF production process. However, due to the residence time in the cyclones, its measurement is performed with a delay of $t_{d}=25 \mathrm{~s}$. Moreover, the air temperature at the tube inlet $T_{\text {in }}$ is required to be within limits due to material and operation constraints.

\section{A. Grey-box model}

Detailed first-principle models of MDF dryers involve partial differential equations (PDE) [23], which are computationally expensive to solve in online MPC optimizations. Moreover, in our case the residence time of the fibers inside the dryer tube is found to be less than the sampling time of the data acquisition system $\left(t_{s}=5 \mathrm{~s}\right.$.), so no information of the drying dynamics inside the tube can be fitted to experimental data. Therefore, given these limitations, we proposed a grey-box lumped-parameter model for control purposes. The model backbone bases on mass and energy balances. For the mixture chamber we have:

$$
\begin{gathered}
F_{\text {in }}=F_{\text {amb }}+F_{\text {hot }} \\
F_{\text {in }} H\left(T_{\text {in }}, W\right)=F_{a m b} H\left(T_{a m b}, W\right)+F_{\text {hot }} H\left(T_{\text {hot }}, W\right)
\end{gathered}
$$

Where $F$ represents the humid air flows in $\mathrm{kg} / \mathrm{s}, H(\cdot, \cdot)$ is the specific enthalpy function in $\mathrm{J} / \mathrm{kg}$ dependent on the air tempera- 
ture $T$ in ${ }^{\circ} \mathrm{C}$ and the specific humidity $W$ (mass of water relative to the total inlet air mass) by the formula:

$$
H(T, W)=1.006 T+(2490+1.86 T) W
$$

And for the dryer tube we have:

$$
\begin{gathered}
F_{\text {in }}+E=F_{\text {out }} \\
q_{f} X_{0}=E+\left(q_{f}-E\right) X \\
F_{\text {in }} H\left(T_{\text {in }}, W\right)+q_{f} C_{p w} T_{\text {fin }}+\left(T_{\text {fin }}-T_{\text {fout }}\right) q_{f} C_{p f}= \\
Q_{T}+F_{\text {out }} H\left(T_{\text {out }}, W_{\text {out }}\right)+q_{f} X C_{p w} T_{\text {fout }}
\end{gathered}
$$

Where $E$ is the evaporated water flow in $\mathrm{kg} / \mathrm{s}, q_{f}$ is the inlet fiber flow in $\mathrm{kg} / \mathrm{s}, T_{\text {fin }}$ and $T_{\text {fout }}$ are the fiber inlet and outlet temperatures, $X_{0}$ and $X$ are the inlet and outlet specific water contents in the fiber respectively, $C_{p w}$ and $C_{p f}$ are the water and dry wood specific heats respectively, and finally $Q_{T}$ is the heat loss to the tube computed by:

$$
Q_{T}=\Delta T_{M} \cdot A \cdot U
$$

Here $U$ is the heat-transfer convection coefficient under turbulent flow ${ }^{2}, A$ is the exchange surface and $\Delta T_{M}$ is the temperature difference between the internal flow (air plus fiber) and the tube averaged ones (to avoid PDEs). The evolution of the tube temperature $T_{t}$ follows the dynamics:

$$
\left(m_{t} C_{p t}+m_{w} C_{p l}\right) \dot{T}_{t}=\Delta T_{M} A \cdot U-\frac{\left(T_{t}-T_{w}\right) A_{e} \cdot k}{e_{w}+e_{t}}
$$

Where $m_{t}, e_{t}, m_{w}$ and $e_{w}$ are the mass and width of the steel tube and mineral-wool covering respectively. $T_{w}$ is the average temperature of the covering. Moreover, $T_{w}$ is related with $T_{a m b}$ by the heat loss from the tube to the ambient:

$$
\frac{\left(T_{t}-T_{w}\right) \cdot A_{e} \cdot k}{e_{w}+e_{t}}=\left(T_{w}-T_{a m b}\right) \cdot A_{e} \cdot U_{e}
$$

Here $k$ is the overall thermal conduction coefficient computed from the respective tube wall materials, and $U_{e}$ is the heat-transfer convection coefficient under laminar flow. The cyclone stage is modeled by first-order dynamics with delay:

$$
\tau \dot{X}_{f}=X\left(t-t_{d}\right)-X_{f}
$$

Where $X_{f}$ is the final fiber humidity (measurable), $\tau$ is an experimentally identified time constant and $t_{d}$ is the delay.

The model is completed with experimental patterns obtained from input-output data recorded from the actual plant. Essentially we followed the methodology proposed in [24], where estimates over time for some internal (unmeasured) variables $z$ are computed and then regression constraints $z(t, u, y)$ are fitted for them.

In this way, experimental equations have been obtained for the total air flow $F_{\text {in }}$ and for the relationship between hot and cold flows $F_{h o t}, F_{a m b}$, with respect to flaps openness.

$$
\begin{gathered}
F_{\text {in }}=K_{f}\left(1-\exp \left(-7.1\left(a_{c}+a_{f}\right)\right)\right) \\
\frac{F_{\text {hot }}}{F_{a m b}}=K_{T} \sqrt{\frac{a_{c}}{a_{f}}}
\end{gathered}
$$

\footnotetext{
${ }^{2}$ Experimental formulas for it can be found in the related literature.
}

Where $K_{f}$ and $K_{T}$ are positive experimental parameters. Finally, a linear relationship with the difference between the dry bulb and wet bulb air temperatures at the dryer outlet has been found here to predict accurate enough the outlet fiber humidity:

$$
\begin{gathered}
X=\gamma_{1}-\gamma_{2} \cdot\left(T_{o u t}-T_{w b}\right) \\
\gamma_{2}=0.9 \gamma_{1}+0.13
\end{gathered}
$$

Where $\gamma_{1}$ is again an experimental parameter to be obtained from data and $T_{w b}$ is the wet-bulb temperature calculated with $T_{\text {out }}$ and the specific humidity $W_{\text {out }}[25]$.

The model gets the following control inputs $u=\left[a_{c}, a_{f}\right]$ and disturbances $d=\left[q_{f}, T_{a m b}, W, T_{h o t}, X_{0}\right]$ to compute the outputs $y=\left[X_{f}, T_{\text {in }}, F_{\text {in }}, T_{\text {out }}\right]$. Among them, only real-time measurements for the inlet fiber humidity $X_{0}$ are not available. Hence, $X_{0}$ and the model parameters $\gamma_{1}, K_{f}$ and $K_{T}$ are selected to be estimated online by a moving-horizon estimator (MHE), following the usual offset-free MPC implementation [26] [8].

\section{B. Nonlinear MPC setup}

Based on the previous grey-box model, a nonlinear MPC setup is proposed in accordance with the control architecture of Fig. 2. For an efficient implementation, the dryer dynamics in (10) and (12) are discretized by orthogonal collocation using 2degree interpolating polynomials and 5 seconds length finite elements [27]. Moreover, to enhance the resolution speed, a proper warm start is provided to IPOPT for each execution in a receding-horizon strategy (see Section IV for details).

Hence, assuming that the system output in steady state $y_{s}$ univocally defines an equilibrium point $\left(x_{s}, u_{s}\right)$ and that the linearized system at each feasible equilibrium is controllable, the following nonlinear MPC problem is set up:

$$
\begin{aligned}
\min _{\theta} J:= & \sum_{t=0}^{H} w_{y}\left|y(t)-y_{S}\right|_{2}^{2}+\sum_{t=0}^{H_{C}-1} w_{u}|\Delta u|_{2}^{2}+w_{s p}\left|X_{f S}-X_{f R}\right|_{1} \\
\text { s.t.: } & (4)-(16), \quad 160<T_{i n}(t), T_{i n S}<210, \\
& 5<u_{i}(t), u_{S}<100, \Delta u^{2} \leq 5^{2}, u(t) \equiv u_{S} \forall t \geq H_{C} \\
& f\left(x_{S}, u_{S}\right)=0, h\left(x_{S}, u_{S}\right)=0, y_{S}=C \cdot x_{S}
\end{aligned}
$$

Where $H$ denotes the prediction horizon, $H_{C}<H$ is the control horizon (so that the controller output remains constant in a suitable steady state $u_{S}$ after $\left.H_{C}\right), \theta=\left[u(t), u_{S}, x_{S}\right]$ are decision variables, $\Delta u=u(t)-u(t-1),\left[w_{y}, w_{u}, w_{s p}\right]$ are the weighting factors and constraints $f(\cdot)$ and $h(\cdot)$ and are the model equations in steady state, i.e. $\dot{X}_{f}=0, \dot{T}_{t}=0$.

Note that artificial setpoints $y_{S}=\left[X_{f S}, T_{i n s}\right]$ are introduced to guarantee the feasibility of the MPC [28]. Hence, the fiber humidity will eventually reach the user-defined set point $X_{f R}$ if $w_{s p}$ in the offset cost is high enough.

\section{Static friction in the air flaps}

The MPC optimization (17) only considers actuators with maximum rate constraints, but experimental data recorded from the plant reveals that the pneumatic mechanism to control the air flaps $a_{c}$ and $a_{f}$ presents stiction. Analyzing the recorded data, we can roughly represent this phenomenon by (1), with parameter $\mathrm{f}_{\mathrm{s}}=0.5$ (in $\%$ of the total flap openness). 


\section{PRACTICAL Stiction COMPENSATION MEthods}

Once the options that involve sampling at higher frequencies are discarded due to computational limitations in MPC and the approach in [8] is unsuccessful because of the reasons discussed in Section I, we came up with the following two modelfree "practical" solutions to handle the flaps stiction issues.

\section{A. Windup compensator}

The first one is a simple modification of the integral-term compensator but, inspired in [13], the compensator output is reset to zero when $u_{c}=u_{a}$ (Fig. 3). In this way, the local compensator does not modify the MPC output when stiction is not active in the actuator.

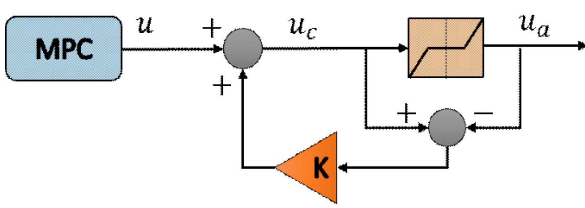

Figure 3. MPC with proposed windup compensator.

The idea behind is that the integral of the actuation error $e=u-u_{a}$ over a time horizon represents somehow the actuation energy that the MPC should deliver to the plant in order to drive its state to the optimal. Therefore, what we propose with the windup compensator is to trigger a pulse larger than $f_{s}$ such that its energy during $T_{s}$ seconds approaches the integral of the error over the previous samples where the actuator is stuck, thereby moving away from the point with steady-state error. This allows the MPC to effectively apply (or at least approach) the optimal $u_{S}$ in subsequent samples. The implementable discrete-time form of the proposed compensator in Fig. 3 is

$$
u_{c}(t+1)=u(t)+K \cdot\left(u_{c}(t)-u_{a}(t)\right)
$$

and $0<K \leq 1$ is the tuning parameter.

Moreover, providing the MPC optimization with information about the compensator behavior can lead to improved control performance. Using the smoothing function (3), the MPC problem (17) is extended with the following constraints ${ }^{3}$ :

$$
\begin{gathered}
\eta=\frac{1}{2}\left(\tanh \left(\left(u_{0}-\chi_{0}+\mathrm{f}_{\mathrm{s}}\right) \tau\right)+\tanh \left(\left(\mathrm{f}_{\mathrm{s}}-u_{0}+\chi_{0}\right) \tau\right)\right) \\
\chi=\eta \cdot\left(u+K \cdot\left(u_{0}-\chi_{0}\right)\right)+(1-\eta) \cdot u
\end{gathered}
$$

Where the new variables $\chi$ are actually replacing $u$ in the plant model equations, and $\chi_{0}, u_{0}$ are values from the previous instant, accomplishing with the updating rules:

$$
u_{0}=\chi ; \quad \chi_{0}=(1-\eta) \cdot \chi
$$

Remark. Note that, in contrast to (3), (19) does not impose stiff constraints on the optimization, because $\chi_{0}$ and $u_{0}$ are values computed at the previous instant. As payback, the above formulation does not allow us to introduce the stiction model in the MPC but just the windup compensator, i.e. the MPC knows about the existence of $u_{c}$ but does not know that some of its values along the prediction horizon won't reach the plant.

\footnotetext{
${ }^{3}$ Expressions (19)-(21) abuse vector notation for simplicity, but they are stated component wise in the MPC optimization problem.
}

\section{B. Deadband penalty}

In contrast to the previous method, here we are not including an external compensator, but the aim is to tell somehow the MPC that control outputs within $|\Delta u|<\mathrm{f}_{\mathrm{s}}$ should not be delivered because they won't be applied due to actuator stiction. The aim is the same as in [8] and so, inspired on it, we make use again of the smoothing function (3) but without involving any stiff constraint. The idea is, instead of "strictly" imposing constraint (2)-(3), to include it as an additional penalty term in the objective function $J$ of problem (17), so that selecting control actions within the deadband is more expensive. Hence, the proposed additional penalty term $J_{p}$ is:

$$
\begin{gathered}
J_{p}:=\sum_{t=0}^{H_{C}-1} w_{u} \cdot L(t) \\
L=\mu \cdot\left(\tanh \left(\left(\Delta u^{2}-\epsilon\right) \tau_{1}\right)+\tanh \left(\left(\mathrm{f}_{\mathrm{s}}^{2}-\Delta u^{2}\right) \tau_{2}\right)\right)
\end{gathered}
$$

Where $\left[\tau_{1}, \tau_{2}, \epsilon\right] \in \mathbb{R}^{+}$are design parameters and $\mu>0$ is the tuning parameter for the penalty function, whose meaning is graphically explained in Fig. 4.

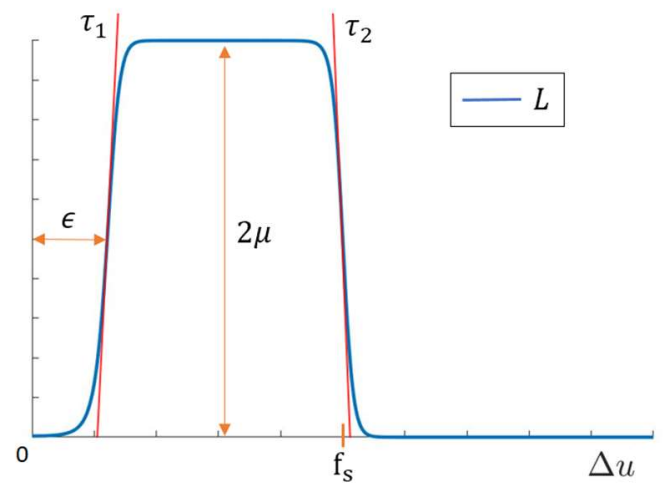

Figure 4. Deadband penalty.

Qualitatively, $\tau_{1}$ and $\tau_{2}$ define the slopes of the "step" edges (higher slopes approximate discrete decisions but are deleterious for gradient-based optimization) and $\epsilon$ defines the small tolerance to allow $\Delta u=0$. These are fixed a priori by the designer. Only $\mu$ is left for further tuning, defining the height of the step. The reason the height is the more relevant tuning parameter affecting performance is graphically given in Fig. 5, where the deadband penalty (22) is combined with the usual input rate quadratic penalty in (17) as follows:

$$
J_{u}:=J_{p}+\sum_{t=0}^{H_{C}-1} w_{u}|\Delta u|_{2}^{2}
$$

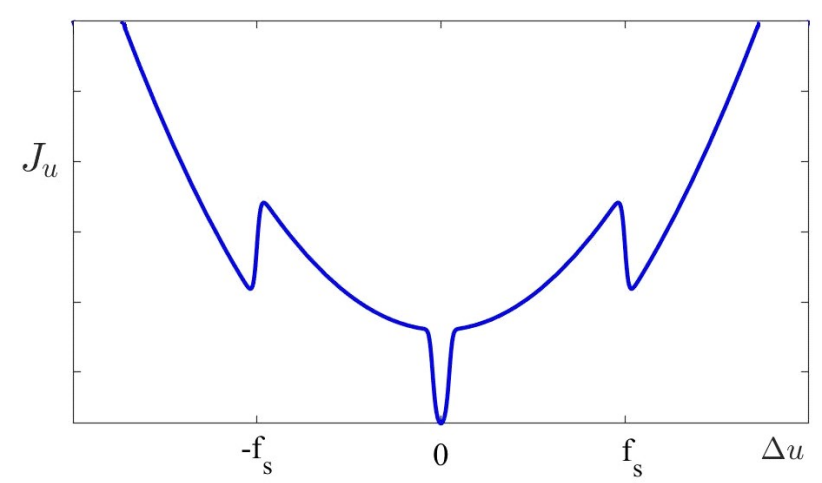

Figure 5. Modified input rate penalty $J_{u}$. 
This combination creates an additional penalty if the solver selects to apply $\epsilon^{2} \leq \Delta_{u}^{2} \leq \mathrm{f}_{\mathrm{s}}^{2}$, so it will be usually avoided. Of course, this is neither a strict restriction nor guarantees global optimality, as $J_{u}$ is noncovex. Nevertheless, (17) was already nonconvex due to plant model nonlinearities, so no specially harmful solutions are found if $\mu$ is suitably chosen.

\section{Simulation TeSts}

Some performance tests were run to quantify the benefits (performance loss w.r.t the perfect actuator) of the proposed approaches. The tests were done against a plant simulation that uses a PDE model of the drying section [23]:

$$
\frac{\partial X}{\partial l}=v\left(\frac{2}{L_{f}}+\frac{4}{d_{f}}\right) U \frac{T_{d b}-T_{w b}}{\Delta H_{w} q_{f}}
$$

Where $l$ is the spatial variable along the tube, $v$ is a parameter which depends on the drying regime, $\left(L_{f}, d_{f}\right)$ are the length and diameter of the fibers, $U$ is the heat-transfer coefficient, $\left(T_{d b}, T_{w b}\right)$ are the air dry-bulb and wet-bulb temperatures and $\Delta H_{w}$ is the water vaporization heat. This creates a strong plantmodel mismatch, both structural and parametric.

In a first test, induced step changes of $3 \%$ in the setpoint $X_{f R} ; 15^{\circ} \mathrm{C}$ in the gasses temperature $T_{h o} ; 3 \mathrm{~T} / \mathrm{h}$ in the fiber flow $q_{f}$ (usual product changeover), $5 \%$ in the fiber inlet humidity $X_{0}$ and $12.5 \%$ in the fiber diameter $d_{f}$ are introduced sequentially every 300 s. Fig. 6 shows the evolution of the controlled humidity $X_{f}$ over time for the following scenarios: a) perfect actuator; b) sticky actuator with no compensation; c) windup compensator; and d) deadband penalty.

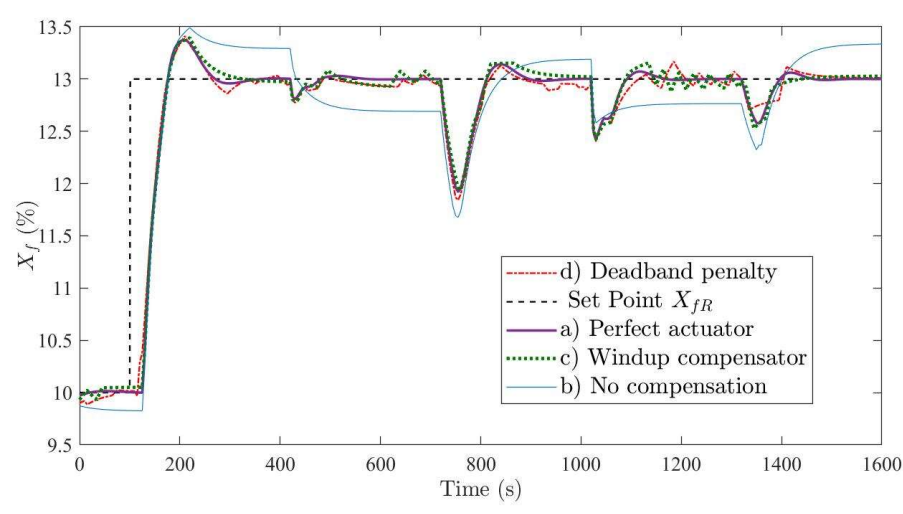

Figure 6. Evolution of the output fiber humidity $X_{f}$.

It is shown that the tested disturbances are totally rejected in the case of control under perfect actuator, with the dynamic response defined by the MPC weighting factors (fixed for all scenarios). Of course, the MPC without any compensation leads to different levels of steady-state errors. On the contrary, the evolution with the two proposed compensation methods approach the response obtained under the assumption of perfect actuator in place, although a small oscillatory behavior (typical from former linear compensators) has been detected between $t=1100$ to $t=1300 \mathrm{~s}$. Note that the proposed practical approaches do not guarantee asymptotic stability but just practical one, so small oscillatory behavior can appear when the plant becomes less controllable. For instance, when the gasses temperature $T_{h o t}$ increases significantly and the fiber diameter is small, so the smallest achievable flap movement (because of stiction) has a bigger impact on the final fiber humidity.

Table I shows a comparison of the above responses under several standard performance measures: the integral square error w.r.t. the setpoint (ISE), the integral absolute error of the humidity outside confidence bands of $\pm 5 \%$ (IAE $5 \%$ ) and $\pm 3 \%$ (IAE 3\%) around the setpoint, the settling time at 95\% of the setpoint step (Te 95\%), the average computational time to solve the MPC optimization (CPU) and the actual number of flaps movements during the whole test ( $a_{c}$ Mov, $a_{f}$ Mov).

TABLE I. PERFORMANCE UNDER STEP-TYPE DISTURBANCES

\begin{tabular}{|l|c|c|c|c|c|}
\cline { 2 - 6 } \multicolumn{1}{c|}{} & $\begin{array}{c}\text { Perfect } \\
\text { Actuator }\end{array}$ & $\begin{array}{c}\text { No } \\
\text { Comp. }\end{array}$ & $\begin{array}{c}\text { External } \\
\text { Windup }\end{array}$ & $\begin{array}{c}\text { MPC } \\
\text { Windup }\end{array}$ & $\begin{array}{c}\text { DB } \\
\text { Penalty }\end{array}$ \\
\hline ISE & 399.67 & 553.96 & 400.76 & 392.55 & 372.52 \\
\hline IAE 5\% & 120.43 & 143.87 & 121.2 & 116.76 & 115.87 \\
\hline IAE 3\% & 274.79 & 340.55 & 278.28 & 270.25 & 264.48 \\
\hline Te 95\% & $144 \mathrm{~s}$ & - & $147 \mathrm{~s}$ & $154 \mathrm{~s}$ & $145 \mathrm{~s}$ \\
\hline CPU & $0.68 \mathrm{~s}$ & $0.34 \mathrm{~s}$ & $0.41 \mathrm{~s}$ & $0.53 \mathrm{~s}$ & $1.44 \mathrm{~s}$ \\
\hline $\boldsymbol{a}_{\boldsymbol{c}}$ Mov & 103 & 21 & 43 & 47 & 59 \\
\hline $\boldsymbol{a}_{\boldsymbol{f}}$ Mov & 103 & 9 & 26 & 21 & 33 \\
\hline
\end{tabular}

Two sets of results are presented in Table I for the windup approach (columns 4 and 5). Values in the MPC Windup column correspond to the whole approach in Section III-A whereas, for completeness, External Windup refers to the MPCunaware basic implementation of the windup compensator depicted in Fig. 3, i.e. (18). The goal of showing this difference was just to stress out the relative importance of making the MPC aware of the external compensator.

Note that both proposed compensation approaches not only improve performance over the stiction-unaware controller, but they even outperform the situation with perfect actuator in place. Moreover, this performance improvement over the perfect situation is obtained with a lower number of flaps movements, which reduces actuator wear. Though this may be a result of share luck, because the MPC could become more aggressive by a different choice of its weighting factors, retuning the main controller is not our intention. The choice of the tuning parameters for the compensation strategies was $K=0.8$ for the windup compensator and $\mu=0.02 \quad\left(\tau_{1}=2000\right.$, $\left.\tau_{2}=100\right)$ for the deadband penalty, both chosen via simple trial and error and visual inspection.

TABLE II. PERFORMANCE UNDER PROCESS NOISE

\begin{tabular}{|l|c|c|c|c|}
\cline { 2 - 5 } \multicolumn{1}{c|}{} & $\begin{array}{c}\text { Perfect } \\
\text { Actuator }\end{array}$ & $\begin{array}{c}\text { No } \\
\text { Comp. }\end{array}$ & $\begin{array}{c}\text { MPC } \\
\text { Windup }\end{array}$ & $\begin{array}{c}\text { DB } \\
\text { Penalty }\end{array}$ \\
\hline ISE & 96.5 & 130.14 & 99.56 & 94.4 \\
\hline IAE 5\% & 5.8 & 5.63 & 7.17 & 1.38 \\
\hline IAE 3\% & 50.78 & 61.16 & 55.07 & 37.03 \\
\hline $\boldsymbol{a}_{\boldsymbol{c}}$ Mov & 104 & 31 & 62 & 42 \\
\hline $\boldsymbol{a}_{\boldsymbol{f}}$ Mov & 103 & 10 & 27 & 15 \\
\hline
\end{tabular}

In a second test, additional random signals of suitable frequencies and powers have been added to the sensors as well as to the gasses temperature $T_{h o t}$ and to the fiber humidity $X$ be- 
fore the cyclones (unmeasured), in order to simulate measurement noise and unexpected time-varying process disturbances similar to the ones observed in the actual facility. In this case, the set point is constant but the all induced step disturbances were kept. Table II shows the performance values got for this test and Fig. 7 depicts the dryer time responses. The results in presence of random process noise show that the deadband penalty approach clearly outperforms the windup compensation as well as the situation with perfect actuator, both in error reduction (ISE, IAE) and in actuator wear.

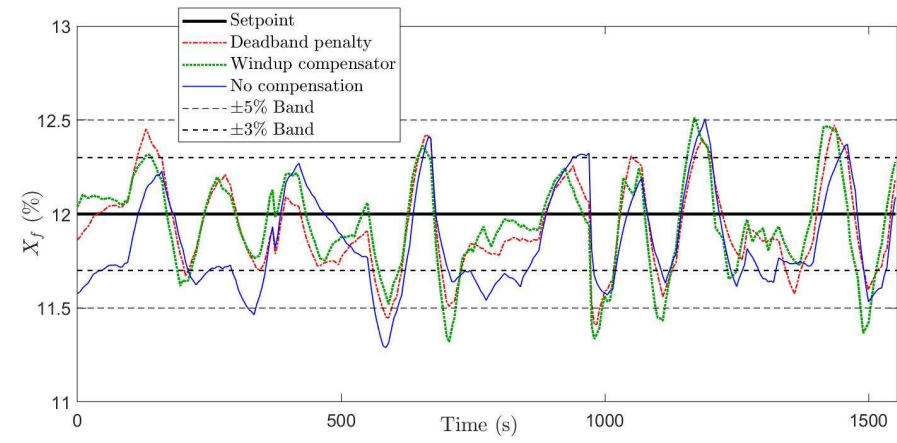

Figure 7. Evolution of the output fiber humidity $X_{f}$.

In all cases, the following sequence of flap inputs is proposed as warm start for the nonlinear MPC optimization:

$$
\begin{aligned}
& u(1)= \begin{cases}u(0)+a \cdot \mathrm{f}_{\mathrm{s}} & \text { if } u(0) \geq u_{S}^{*} \\
u(0)-a \cdot \mathrm{f}_{\mathrm{s}} & \text { if } u(0)<u_{S}^{*}\end{cases} \\
& u(t)=u(t)^{*} \forall t>1
\end{aligned}
$$

Where $a>2, u(0)$ are the last applied actions, and notation $u^{*}$ stands for the optimal values of $u$ in the previous run.

\section{CONCLUSION}

We proposed two practical strategies for stiction compensation that are suitable to be combined with general nonlinear MPC for MIMO systems. Both approaches were tested in an industrial case with successful results, and keeping similar resolution times of the original MPC implementation.

Despite their simplicity and clear limitations, the proposed stiction compensation approaches are proven to provide significant improved control performance with reduced actuator wear in our case study, even in presence of the strong plant-model mismatch and process disturbances. Furthermore, the deadband penalty approach seems to perform better than the windup compensator in situations with significant time-varying disturbances and measurement noise. However, the adequate selection of the compensator parameters as well as the compensation problem itself remains open for further systematic research.

\section{REFERENCES}

[1] T. Hägglund, "Automatic on-line estimation of backlash in control loops," Journal of Process Control, vol. 17 (6), pp. 489-499, 2007.

[2] M.S. Choudhury, S.L. Shah, N. Thornhill and D.S. Shook, "Automatic detection and quantification of stiction in control valves," Control Engineering Practice, vol. 14 (12), pp. 1395-1412, 2006.

[3] X. Zhao and Y. Tan, "Neural network based identification of Preisach type hysteresis in piezoelectric actuator using hysteretic operator," Sensors and Actuators A: Physical, vol. 126 (2), pp. 306-311, 2006.

[4] M.C. Rodríguez Liñán, "New control design and analysis techniques for plants with actuator nonlinearities," Ph.D. dissertation, School of Electrical and Electronic Eng., University of Manchester, UK, 2013.
[5] R.B. di Capaci and C. Scali, "Review and comparison of techniques of analysis of valve stiction: From modeling to smart diagnosis," Chem. Engineering Research and Design, vol. 130, pp. 230-265, 2018.

[6] H. Durand, R. Parker, A. Alanqar and P.D. Christofides, "Elucidating and handling effects of valve-induced nonlinearities in industrial feedback control loops," Computers \& Chemical Eng., In Press, 2017.

[7] P. Mishra, V. Kumar and K.P.S. Rana, "An online tuned novel nonlinear PI controller for stiction compensation in pneumatic control valves," ISA Transactions, vol. 58, pp. 434-445, 2015.

[8] R.B. di Capaci, M. Vaccari and G. Pannocchia, "A valve stiction tolerant formulation of MPC for industrial processes," IFAC-PapersOnLine, vol. 50 (1), pp. 9044-9049, 2017.

[9] M. Ale Mohammad and B. Huang, "Compensation of control valve stiction through controller tuning," Journal of Process Control, vol. 22, pp. 1800-1819, 2012.

[10] T. Hägglund, "A friction compensator for pneumatic control valves," Journal of Process Control, vol. 12, pp. 897-904, 2002.

[11] R. Srinivasan and R. Rengaswamy, "Approaches for efficient stiction compensation in the process control valves," Computers \& Chemical Engineering, vol. 32, pp. 218-229, 2008 .

[12] L. Z. X. Ivan and S. Lakshminarayanan, "A new unified approach to valve stiction quantification and compensation," Industrial \& Engineering Chemistry Research, vol. 48, pp. 3474-3483, 2009.

[13] M.A. de Souza L. Cuadros, C.J. Munaro and S. Munareto, "Novel Model-Free Approach for Stiction Compensation in Control Valves," Industrial \& Eng. Chemistry Research, vol. 51 (25), pp. 8465-8476, 2012.

[14] N. F. Thornhill and A. Horch, "Advances and new directions in plantwide disturbance detection and diagnosis," Control Engineering Practice, vol. 15, pp. 1196-1206, 2007

[15] Q.P. He, J. Wang, M. Pottmann, and S.J. Qin, "A curve fitting method for detecting valve stiction in oscillating control loops," Industrial \& Engineering Chemistry Research, vol. 46, pp. 4549-4560, 2007.

[16] Q.P. He and J. Wang, "Valve stiction modeling: First-principles vs. datadrive approaches," in Proc. of the $7^{\text {th }}$ American Control Conf., Baltimore, 2010, pp. 3777-3782.

[17] H. Zabiri and Y. Samyudia, "MIQP-Based MPC in the Presence of Control Valve Stiction," Chem. Prod. \& Process Model., vol. 4(3), 2009.

[18] H. Durand and P.D. Christofides, "Actuator stiction compensation via model predictive control for nonlinear processes," AIChE Journal, vol. 62 (6), pp. 2004-2023, 2016.

[19] H. Durand and P.D. Christofides, "Empirical modeling of control valve layer with application to model predictive control-based stiction compensation," IFAC-PapersOnLine, vol. 49 (18), pp. 41-46, 2016.

[20] H. Zabiri and Y. Samyudia, "A hybrid formulation and design of model predictive control for systems under actuator saturation and backlash," Journal of Process Control, vol. 16, pp. 693-709, 2006.

[21] J. Novak and P. Chalupa, "Compensation of valve deadzone using mixed integer predictive control," in Proc. of the $31^{\text {st }}$ European Conference on Modelling and Simulation ECMS 2017, pp. 379-383, Budapest, 2017.

[22] J. Andersson, J. Åkesson, and M. Diehl, "CasADi: A Symbolic Package for Automatic Differentiation and Optimal Control," in Recent Advances in Algorithmic Differentiation, Springer, 2012, pp. 297-307.

[23] O. Melander and A. Rasmuson, "Mathematical modeling of heat, mass, and momentum transport in MDF fiber drying," Drying Technology, vol. 29 (1), pp. 64-72, 2010.

[24] C. de Prada, D. Hose, G. Gutierrez, and J.L. Pitarch, "Developing greybox dynamic process models," IFAC-PapersOnLine, vol. 51 (2), pp. 523-528, 2018.

[25] R. Stull, "Wet-bulb temperature from relative humidity and air temperature," Journal of Applied Meteorology and Climatology, vol. 50 (11), pp. 2267-2269, 2011.

[26] G. Pannocchia, M. Gabiccini and A. Artoni, "Offset-free MPC explained: novelties, subtleties, and applications," IFAC-PapersOnLine, vol. 48 (23), pp. 342-351, 2015.

[27] L.T. Biegler, Nonlinear programming: concepts, algorithms, and applications to chemical processes, SIAM Series on Optimization, 2010.

[28] D. Limon, A. Ferramosca, I. Alvarado, and T. Alamo, "Nonlinear MPC for tracking piece-wise constant reference signals," IEEE Transactions on Automatic Control, DOI:10.1109/TAC.2018.2798803 In Press, 2018. 